Arts

et Savoirs
Arts et Savoirs

12 | 2019

Révolution et évolution

\title{
Évolutions ou révolutions : le rôle de l'événement chez Flaubert
}

Evolution or Revolution : the Meaning of Event in Flaubert's Work

Juliette Azoulai

\section{OpenEdition}

Journals

Édition électronique

URL : http://journals.openedition.org/aes/2209

DOI : 10.4000/aes.2209

ISSN : 2258-093X

Éditeur

Laboratoire LISAA

Référence électronique

Juliette Azoulai, «Évolutions ou révolutions : le rôle de l'événement chez Flaubert 》, Arts et Savoirs [En ligne], 12 | 2019, mis en ligne le 06 février 2020, consulté le 02 mars 2020. URL : http://

journals.openedition.org/aes/2209; DOI : 10.4000/aes.2209

Ce document a été généré automatiquement le 2 mars 2020.

Centre de recherche LISAA (Littératures SAvoirs et Arts) 


\title{
Évolutions ou révolutions : le rôle de l'événement chez Flaubert
}

\author{
Evolution or Revolution : the Meaning of Event in Flaubert's Work
}

\author{
Juliette Azoulai
}

1 Selon Gérard Genette, « dès qu'il y a acte ou événement, fût-il unique, il y a histoire, car il y a transformation, passage d'un état antérieur à un état ultérieur et résultant ${ }^{1}$. Le récit, dans sa forme minimale, suppose donc l'événement, en tant que celui-ci implique une différence entre un avant et un après. Or toute l'originalité de Flaubert, qui admettait lui-même que « le récit est une chose qui [lui] est très fastidieuse ${ }^{2}$, consiste précisément dans l'amoindrissement de l'événement et de l'action romanesques; comme le résume Jean Rousset: «ce n'est pas l'événement qui se contracte sous la main de Flaubert, mais ce qu'il y a entre les événements, ces étendues stagnantes où tout mouvement s'immobilise $»^{3}$. Comme la critique l'a amplement montré, Flaubert, écrivain de la réaction plus que de l'action, de l'immobile plus que du mouvement, invente un nouveau type de récit romanesque, réfractaire à l'événement ${ }^{4}$. Ce lieu commun esthétique nous paraît cependant mériter un approfondissement conceptuel : qu'est-ce que l'œuvre de Flaubert fait au concept d'événement et en quoi reconfigure-telle les formes du dynamisme et de la temporalité romanesques?

2 Nous tenterons de comprendre le mode de temporalité qu'invente la poétique flaubertienne de l'événement à la lumière du contexte intellectuel du XIX ${ }^{e}$ siècle, qui est celui d'une complexification et d'un entrelacement des concepts d'évolution et de révolution : au début du siècle, avec le catastrophisme de Cuvier, la révolution, notion historique, devient applicable à la nature (à la géologie et à la biologie) ; avec Geoffroy Saint Hilaire, la notion d'évolution, qui désignait, dans l'embryologie du XVII et du XVIII siècles, le développement d'un germe préformé, devient un principe de compréhension diachronique de la variété des espèces et, avec Spencer, passe dans le champ de la sociologie et de l'histoire. Les vicissitudes de ces deux concepts - évolution et révolution - conduisent à des débats, en sciences humaines et en sciences naturelles, quant à la validité des conceptions continuiste et discontinuiste du temps et quant à l'existence ou non d'événements exceptionnels (fondateurs et destructeurs) qui 
rythmeraient le temps historique et le temps naturel. Entre géologie catastrophiste et géologie uniformitariste, entre biologie fixiste et biologie transformiste, entre historiographie événementielle et historiographie de la longue durée ${ }^{5}$, entre immanentisme et interventionnisme socio-politiques (laisser faire l'évolution ou faire la révolution?), les conceptions évolutionnistes et révolutionnaires du temps induisent des controverses dans de multiples champs disciplinaires, qui souvent s'interpénètrent.

Afin de saisir le rôle de l'événement dans la pensée flaubertienne, nous chercherons donc à replacer l'écrivain dans les débats de son temps, en examinant différentes occurrences, dans son œuvre, du mot ou de l'idée d'événement - qu'elles relèvent de la vie individuelle ou collective. Il s'agira en somme de suivre à la trace une pensée qui tout à la fois récuse, démystifie et redéfinit ce qui fait événement dans la Nature, mais aussi dans l'histoire collective et dans l'existence individuelle.

\section{Révolutions féeriques : désenchanter l'événement}

4 La notion d'événement constitue d'abord pour les personnages un objet de croyance, que la narration fait apparaître comme un mythe. Emma en particulier semble hantée par la superstition de l'événement. Aussi, à Tostes, appelle-t-elle de ses vœux un hypothétique événement, susceptible, pour le meilleur ou pour le pire, de briser le cercle infernal de l'ennui :

Au fond de son âme, cependant, elle attendait un événement. Comme les matelots en détresse, elle promenait sur la solitude de sa vie des yeux désespérés, cherchant au loin quelque voile blanche dans les brumes de l'horizon. Elle ne savait pas quel serait ce hasard, le vent qui le pousserait jusqu'à elle, vers quel rivage il la mènerait, s'il était chaloupe ou vaisseau à trois ponts, chargé d'angoisses ou plein de félicités jusqu'aux sabords. Mais, chaque matin, à son réveil, elle l'espérait pour la journée, et elle écoutait tous les bruits, se levait en sursaut, s'étonnait qu'il ne vînt pas ; puis, au coucher du soleil, toujours plus triste, désirait être au lendemain. ${ }^{6}$

5 L'espoir d'Emma consiste dans l'attente d'un événement qui pourrait marquer une rupture, heureuse ou malheureuse, dans la monotone répétition des jours. Mais la déception quotidienne d'un tel espoir se mue bientôt en désespoir : son existence lui apparaît alors comme imperméable à toute aventure; et Emma constate mélancoliquement que littéralement rien ne saurait advenir dans sa vie. L'événement se révèle comme illusoire pour elle, là où pour d'autres il serait réalité :

Après l'ennui de cette déception, son cœur, de nouveau, resta vide, et alors la série des mêmes journées recommença.

Elles allaient donc maintenant se suivre ainsi à la file toujours pareilles, innombrables, et n'apportant rien! Les autres existences, si plates qu'elles fussent, avaient du moins la chance d'un événement. Une aventure amenait parfois des péripéties à l'infini, et le décor changeait. Mais, pour elle, rien n'arrivait, Dieu l'avait voulu! L'avenir était un corridor tout noir, et qui avait au fond sa porte bien fermée.

6 Le modèle de référence d'Emma pour penser l'événement semble être un modèle théâtral, comme l'indique la mention des décors changeants. Mais au lieu d'un Deus ex machina qui viendrait changer la face de son existence, Emma doit reconnaître que c'est plutôt un malin génie qui s'est plu à l'enfermer dans le piège fatal d'une vie uniforme. Ce Dieu malveillant pourrait bien être l'auteur ou le narrateur lui-même, qui dépeint en effet le monde de la province normande comme un monde parfaitement immobile, où 
aucun événement, même la fin tragique d'Emma, ne peut venir entraver l'irrémédiable piétinement du temps :

Depuis les événements que l'on va raconter, rien, en effet, n'a changé à Yonville. Le drapeau tricolore de fer-blanc tourne toujours au haut du clocher de l'église; la boutique du marchand de nouveautés agite encore au vent ses deux banderoles d'indienne; les foetus du pharmacien, comme des paquets d'amadou blanc, se pourrissent de plus en plus dans leur alcool bourbeux, et, au-dessus de la grande porte de l'auberge, le vieux lion d'or, déteint par les pluies, montre toujours aux passants sa frisure de caniche. ${ }^{8}$

7 Là où Emma attend un changement à vue, le monde de Yonville se révélera un décor immuable, où la seule perspective d'évolution est celle d'une lente corruption, d'un inexorable pourrissement. Flaubert se situe là dans la continuité de la représentation balzacienne du temps provincial, par opposition au temps parisien : «Si tout arrive à Paris, tout passe en province [...]. »"

8 Face aux aspirations de son héroïne au mouvement et à l'hétérogène, Flaubert oppose un chronotope quasi-statique et homogène, qui pourrait bien être celui du roman par opposition au théâtre, et plus particulièrement à la féerie. En effet dans les brouillons on peut trouver une allusion à ce genre théâtral bien connu de l'auteur :

Les autres existences avaient la chance d'un accident quelconque [...] il se trouvait des péripéties subites, des tragédies - des aventures \& des changements de décors qui vous feraient passer d'une chaumière à un palais. Mais [...] pr elle rien n'arriverait. [...] La bag[uette] d'une fée se fût cassée. ${ }^{10}$

9 Les aventures, les changements de décor et les péripéties subites dont rêve Emma sont intimement liés à un imaginaire féerique, hérité de ses lectures de jeunesse : Emma, trouve-t-on dans les brouillons, avait attendu en vain, petite déjà, "la bonne marraine » des contes de fées qui changeait « les citrouilles en carrosses, les groseilles en rubis $"^{11}$. L'espoir de la métamorphose est en effet au cœur de cette attente de l'événement; mais une fois adulte, Emma prend conscience que son monde n'est aucunement ductile à l'événementialité féerique : « pour soulever un fardeau si lourd la baguette d'une fée eût été un levier trop mince $»^{12}$. Constat éminemment spleenétique, formulé également par Baudelaire dans son poème «L'irréparable » :

Mais mon cœur, que jamais ne visite l'extase,

Est un théâtre où l'on attend

Toujours, toujours en vain, l'Être aux ailes de gaze $!^{13}$

10 L'événement qui serait le coup de baguette magique capable de métamorphoser le hic et nunc de l'existence, de déclencher des péripéties et de faire varier les décors, conformément à la forme ambulatoire de la féerie, ne saurait intervenir dans le monde romanesque et réaliste. Les effets spectaculaires du " théâtre oculaire $»^{14} n$ 'ont plus leur place dans la peinture du monde réel et Flaubert indique ailleurs que le prétendu réalisme d'un Balzac, loin de nous révéler les rouages du monde moderne, n'est qu'une machinerie féerique, inventée de toutes pièces par un auteur pour créer le mirage de l'aventure : «il fallait un événement $»^{15}$, selon l'expression d'Argow le Pirate.

11 En effet, le point de vue cynique de Deslauriers sur le monde, imprégné de littérature balzacienne, est présenté comme celui d'un grand naîf, croyant à la portée miraculeuse de certains événements susceptibles de produire, au terme d'une chaîne de causes et d'effets, des bénéfices incommensurables :

N'ayant jamais vu le monde qu'à travers la fièvre de ses convoitises, il se l'imaginait comme une création artificielle, fonctionnant en vertu de lois mathématiques. Un 
dîner en ville, la rencontre d'un homme en place, le sourire d'une jolie femme pouvaient, par une série d'actions se déduisant les unes des autres, avoir de gigantesques résultats. Certains salons parisiens étaient comme ces machines qui prennent la matière à l'état brut et la rendent centuplée de valeur. Il croyait aux courtisanes conseillant les diplomates, aux riches mariages obtenus par les intrigues, au génie des galériens, aux docilités du hasard sous la main des forts. ${ }^{16}$

Si la métamorphose du destin chez Balzac n'advient qu'à l'issue d'une série causale, et non d'un seul coup de baguette magique, le principe féerique reste identique : la trame du déterminisme se trouve déchirée par le surgissement de l'imprévu; l'événement se produit (le « gigantesque résultat » de l'ascension sociale), en tant qu'il implique, selon les analyses d'Hannah Arendt, une « disparité $»^{17}$ entre l'effet et ses causes : « la matière à l'état brut » a "centuplé de valeur ». Flaubert dénonce bien ici l'artificialité d'une telle conception de l'événement, qui relève d'une représentation passionnelle du monde (vu à travers « la fièvre [des] convoitises »).

Ce modèle d'une temporalité discontinue sera également brocardé dans Bouvard et Pécuchet lorsque les deux héros rêvent à partir de leur lecture du Discours sur les révolutions du globe de Cuvier ${ }^{18}$. Les deux compères se figurent l'histoire de la Terre comme une succession de tableaux disparates. Divers personnages font leur apparition dans des décors chaque fois différents: d'abord un décor «immobile et nu $»^{19}$, dépourvu de vie; puis les plantes dans un univers dominé par les volcans en éruption ; ensuite des animaux marins et des amphibies dans la mer; enfin des grands mammifères sur les continents. L'écriture de Flaubert met en relief la solution de continuité entre les divers tableaux par l'usage du tiret et rapporte cette représentation $d u$ monde au modèle féerique ${ }^{20}$ : «Toutes ces époques avaient été séparées les unes des autres par des cataclysmes, dont le dernier est notre déluge. C'était comme une féerie en plusieurs actes, ayant l'homme pour apothéose. $»^{21}$ Cuvier propose avec l'idée de "révolution du globe " une vision du temps géologique rythmé par des événements destructeurs, les cataclysmes, qui opèrent à chaque fois une tabula rasa, à partir de laquelle un nouveau monde peut renaître et de nouvelles espèces surgir. La pensée cuviériste constitue une hyperbolisation de l'événement comme scission entre un avant et un après, selon le paradigme du soubresaut révolutionnaire. Or cette pensée sera mise à mal, dans l'esprit de Bouvard et Pécuchet, par la découverte des textes transformistes ou pré-évolutionnistes, qui, avant Darwin, se réapproprient le vieil axiome gradualiste de Linné : Natura non facit saltum :

Jamais il n'y eut un cataclysme complet du globe [...]. Les fougères d'autrefois sont identiques aux fougères d'à présent. [...] Les mêmes causes agissent toujours, la Nature ne fait pas de sauts [...].

Cuvier, jusqu'à présent, leur avait apparu dans l'éclat d'une auréole, au sommet d'une science indiscutable. Elle était sapée. La Création n'avait plus la même discipline, et leur respect pour ce grand homme diminua. ${ }^{22}$

Or cette déconstruction de l'événement cataclysmique à la lumière d'une représentation gradualiste du temps façonnait déjà la narration de Madame Bovary, qui ne cessait de mettre en conflit le cours uniforme du temps et les illusions catastrophistes de l'héroïne rêvant de voir sa vie révolutionnée (au sens cuviérien du terme) :

L'amour, croyait-elle, devait arriver tout à coup, avec de grands éclats et des fulgurations, - ouragan des cieux qui tombe sur la vie, la bouleverse, arrache les volontés comme des feuilles et emporte à l'abîme le cœur entier. Elle ne savait pas que, sur la terrasse des maisons, la pluie fait des lacs quand les gouttières sont 
bouchées, et elle fût ainsi demeurée en sa sécurité, lorsqu'elle découvrit subitement une lézarde dans le mur. ${ }^{23}$

15 La passion, loin d'être une apocalypse individuelle, impliquant bouleversement et révélation, est plutôt une accumulation de modifications infimes, obscures et progressives. Évolution, plutôt que révolution, infiltration plutôt qu'ouragan : l'amour n'arrive pas d'un coup ; ce qu'Emma se représente comme une différence de nature au sein de sa propre vie consiste en fait en une différenciation de degré.

Flaubert met en cause la vision d'un temps disloqué et montre qu'une telle représentation est le fait de l'esprit humain, en tant qu'il fantasme de s'approprier le monde. Comme on l'a vu dans Bouvard et Pécuchet, la représentation cuviérienne du temps place l'homme en position éminente: l'homme constitue l'apothéose de la création par périodes. Cette vision téléologique de l'histoire du globe sous-tend certaines représentations que les personnages se font de l'événement. En effet, le désir des personnages flaubertiens de voir advenir un événement qui bouleverserait de fond en comble le cours des choses revient souvent au vœu absurde de conformer les circonstances à leur intérêt particulier, d'aligner les astres en leur faveur. C'est le cas de Cisy dans L'Éducation sentimentale, la veille de son duel tant redouté contre Frédéric :

Il souhaita que Frédéric, pendant la nuit, mourût d'une attaque d'apoplexie, ou qu'une émeute survenant, il y eût le lendemain assez de barricades pour fermer tous les abords du bois de Boulogne, ou qu'un événement empêchât un des témoins de s'y rendre. [...] Il arriva jusqu'à désirer être malade, gravement. ${ }^{24}$

17 De façon similaire, Emma, acculée à la ruine par les nombreuses dettes qu'elle a contractées auprès de l'usurier Lheureux, s'en remet à une Providence qui la sauverait miraculeusement in extremis : «- Advienne que pourra ! se disait-elle. Et puis, qui sait? Pourquoi, d'un moment à l'autre, ne surgirait-il pas un événement extraordinaire? Lheureux même pouvait mourir. ${ }^{25} \mathrm{Ce}$ type d'imagination apparaît cependant sous un jour moins ironique dans un roman comme Salammbô, où le sacré imprègne la vie individuelle et collective : alors que les habitants de Carthage, assiégés par les Barbares, sont menacés de mourir de soif, après le sabotage de l'aqueduc, Hamilcar ne se décourage pas: "Il comptait sur un événement, sur quelque chose de décisif, d'extraordinaire. ${ }^{26}$ De fait, la pluie reviendra sur Carthage, à la suite du sacrifice des enfants à Moloch. Le monde divin est donc susceptible d'intervenir dans le monde humain sous les espèces d'un événement météorologique.

Hélas, l'extraordinaire est réduit à la part congrue dans le monde sécularisé de Madame Bovary. L'invitation à la Vaubyessard constitue l'un de ces événements : « quelque chose d'extraordinaire tomba dans sa vie : elle fut invitée à la Vaubyessard, chez le marquis d'Andervilliers ${ }^{27}$. Cependant si cet événement a bien le caractère d'exceptionnalité qu'en attendait Emma, il ne restera qu'un point dans la durée de son existence. Pire, il fera « un trou dans sa vie, à la manière de ces grandes crevasses, qu'un orage en une seule nuit, creuse quelquefois dans les montagnes ${ }^{28}$. L'événement du bal, loin d'être décisif, d'ouvrir les possibles d'une autre vie, ne formera dans l'existence d'Emma qu'une parenthèse, ce qu'on pourrait paradoxalement définir comme un non-événement. Quelque chose certes est survenu mais pour autant rien n'en est advenu. On retrouve l'image de la catastrophe naturelle (l'orage) mais, contrairement au schéma cuviérien qui voit dans l'événement une puissance à la fois destructrice et créatrice (un nouveau monde peut surgir sur les décombres de l'ancien), Flaubert transforme l'événement en une pure béance (la crevasse dans la montagne), un intervalle vide dans la durée. Emma est redevenue Cendrillon après le $\mathrm{bal}^{29}$, son carrosse est resté citrouille, la 
métamorphose n'a été qu'un mirage éphémère : en effet, si Emma pendant le bal a l'impression d'être brusquement dissociée de son passé («toute son existence antérieure lui parut rentrant en soi comme les cylindres d'une lorgnette et appartenir à quelqu'un d'autre $»^{30}$, indiquent les brouillons), ce présent fulgurant n'ouvre sur aucun avenir : l'événement ne saurait produire l'avènement d'une autre vie. La discontinuité du temps ici n'est donc pas productrice d'un devenir. Cette expérience du trou temporel ${ }^{31}$ se trouvait déjà dans Novembre, où l'extase panthéiste du narrateur se terminait par un retour à la réalité et une forme de dégrisement, très abrupts :

Puis ce fut tout [...] : Je revins le soir chez nous, je repassai par les mêmes chemins, je revis sur le sable la trace de mes pieds et dans l'herbe la place où je m'étais couché, il me sembla que j'avais rêvé. Il y a des jours où l'on a vécu deux existences, la seconde n'est déjà plus que le souvenir de la première, et je m'arrêtais souvent dans mon chemin devant un buisson, devant un arbre, au coin d'une route, comme si là, le matin, il s'était passé quelque événement de ma vie. ${ }^{32}$

Dans un article examinant les moments de "schisme temporel $»^{33}$ dans l'œuvre de Flaubert, Tim Unwin a montré la parenté entre ce passage et le retour d'Emma à Yonville après l'escapade en forêt avec Rodolphe :

Ils s'en revinrent à Yonville, par le même chemin. Ils revirent sur la boue les traces de leurs chevaux, côte à côte, et les mêmes buissons, les mêmes cailloux dans l'herbe. Rien autour d'eux n'avait changé ; et pour elle, cependant, quelque chose était survenu de plus considérable que si les montagnes se fussent déplacées. ${ }^{34}$

20 La discordance entre la permanence du paysage et le sentiment d'un bouleversement existentiel («plus considérable» qu'un bouleversement cosmique) contribue déjà à mettre en doute, dans l'esprit du lecteur, la portée cataclysmique de cet événement qu'Emma voit comme une promesse de métamorphose : "Elle se répétait: "J’ai un amant ! un amant !" se délectant à cette idée comme à celle d'une autre puberté qui lui serait survenue. $»^{35} \mathrm{~L}$ 'héroïne découvrira peu à peu la nature illusoire de cette croyance en la possibilité de transmuter son existence - ce qu'elle analysera plus tard comme « la pourriture instantanée des choses où elle s'appuyait $»^{36}$. Quelque chose s'est bien passé, qui a rompu la trame ordinaire du temps, mais rien n'en est advenu : le décor n'a pas changé ; les montagnes ne se sont pas déplacées.

21 L'illusion féerique de la révolution existentielle a pour pendant dans la sphère collective l'illusion de la révolution politique. Flaubert pourrait ainsi rejoindre le propos de Pierre Nora, lorsque celui-ci affirme : «l'événement c'est le merveilleux des sociétés démocratiques $»^{37}$. Afin de déconstruire le concept de révolution, qu'il juge fantasmagorique, l'écrivain défend à la fin de sa vie une application des théories darwiniennes au champ socio-politique: "Appliquée à l'Histoire [la théorie de l'évolution] met à néant les rêves sociaux $»^{38}$. La société ne saurait changer du jour au lendemain. La vision évolutionniste d'un temps uniforme et continu constitue pour lui un désenchantement salutaire des idéaux socialistes: "La Magie croit aux transformations immédiates par la vertu des formules, absolument comme le Socialisme. Ni l'une ni l'autre ne tiennent compte du temps et de l'Évolution fatale des choses. ${ }^{39}$

L'événement révolutionnaire, en tant qu'il représenterait une déchirure du temps, est pour Flaubert une utopie : l'Histoire doit être envisagée dans la logique continuiste qui est celle de la Nature. Aussi l'écrivain se montre-t-il enclin à pratiquer une lecture social-darwinienne ou socio-biologique de l'Histoire ${ }^{40}$. Loin de vouloir "rend[re] à l'événement sa brutale fraîcheur $»^{41}$, comme entendra le faire Sartre, et au rebours d'un 
Hugo, qui crée en 1848 un journal intitulé L'Événement, Flaubert ne dépeint les événements politiques ni comme des bouleversements collectifs, ni comme des bouleversements intimes. Comme l'ont montré Michel Crouzet ${ }^{42}$ et Victor Brombert ${ }^{43}$, Flaubert, contrairement à la tradition du roman historique, ne cesse dans L'Éducation sentimentale de désolidariser la sphère privée de la sphère collective, en instaurant une cloison entre l'événement politique et les préoccupations des personnages : Frédéric est plus pressé de goûter au fruit défendu avec Mme Arnoux que de faire tomber la « poire [...] mûre $»^{44}$ de la royauté ; en février 1848 , Rosanette et lui seront plus pressés de faire l'amour que la révolution et plus enclins à s'égailler dans la nature de Fontainebleau qu'à s'impliquer dans les journées de Juin 1848. L'événement politique semble ne pas concerner Frédéric. Or, selon la définition du philosophe Claude Romano, ce qui constitue l'essence de l'événement est précisément le fait d'être " toujours adressé $»^{45}$ à quelqu'un, et plus précisément de permettre à celui qui le vit de «se comprendre luimême à partir de [ce] qui [...] lui survient $»^{46}$. L'événement, au sens plein du terme, se distingue alors de ce que Claude Romano appelle le «fait intramondain $»^{47}$, qui ne s'adresse à personne en particulier et se produit indifféremment pour tout témoin. Flaubert semble bien réduire l'événement historique aux dimensions du "fait intramondain", dans la mesure où celui-ci n'instaure chez les sujets aucune modification existentielle.

Le récit flaubertien en ce sens désenchante l'événement, tout en exhibant le cynisme des récitateurs de l'événement : ainsi le journaliste Hussonnet se montre fort peu ému par le spectacle du peuple-roi, lors du sac des Tuileries (« les héros ne sentent pas bon » $\left.{ }^{48}\right)$; pourtant le compte rendu qu'il en fera pour un journal de province n'aura pas du tout la même tonalité :

Hussonnet dit, en bâillant :

- «Il serait temps, peut-être, d'aller instruire les populations !»

Frédéric le suivit à son bureau de correspondance, place de la Bourse; et il se mit à composer pour le journal de Troyes un compte rendu des événements en style lyrique, un véritable morceau, - qu'il signa. ${ }^{49}$

On reconnait au passage le regard, généralement peu amène, que Flaubert porte sur la presse, véritable machine à fabriquer des événements, qui ne sont que de purs artefacts rhétoriques. On rappellera, et c'est tout dire, que Homais est à ses heures un chroniqueur enthousiaste du Fanal de Rouen ${ }^{50}$.

Le dernier degré de dégradation de l'événement historique est atteint dans la conversation, qui, s'emparant des sujets médiatiques, se gargarise de prétendus événements qui sont en réalité le tout-venant d'une actualité insignifiante: «Puis la conversation descendit aux événements contemporains : les mariages espagnols, les dilapidations de Rochefort, le nouveau chapitre de Saint-Denis, ce qui amènerait un redoublement d'impôts. $\|^{51}$

\section{Nature et histoire : " cataclysmes ignorés » et révolutions ignorées}

Cette dissolution du sens de l'événement dans la représentation flaubertienne de l'histoire a déjà été bien soulignée par la critique flaubertienne ${ }^{52}$, mais il s'agit de comprendre à l'aune de quelle représentation du temps se manifeste le caractère dérisoire de l'événementialité politique. 
'on lit l'épisode de Fontainebleau dans L'Éducation sentimentale, il apparait que le temps de la Nature n'est pas un temps continu (temps évolutionniste) qui s'opposerait aux discontinuités du temps historique (temporalité révolutionnaire). Flaubert ne cherche donc pas à déconstruire l'illusion de l'événementialité historique en y opposant la lente progressivité des processus naturels. En effet, ce qui se donne à voir à Frédéric et Rosanette à travers les rochers de Fontainebleau, c'est la trace d'événements cosmiques très anciens et de très grande ampleur :

Mais la furie même de leur chaos fait plutôt rêver à des volcans, à des déluges, aux grands cataclysmes ignorés. Frédéric disait qu'ils étaient là depuis le commencement du monde et resteraient ainsi jusqu'à la fin ; Rosanette détournait la tête, en affirmant que " ça la rendrait folle ».53

L'imaginaire cuviérien de la révolution du globe est ici de nouveau convoqué, mais cette représentation de l'événement cataclysmique universel ne fait pas naître l'idée d'une instabilité du monde, comme on pourrait s'y attendre. Au contraire, elle invite à méditer sur la "permanence des choses ${ }^{54}$, selon une expression des brouillons. La trace des révolutions naturelles rend la temporalité de l'émeute parisienne dérisoire: l'événement géologique et l'événement humain ont lieu dans des échelles de temps tellement incommensurables que le temps de la Terre apparait comme une éternité immobile au regard de l'impermanence des choses humaines. Ainsi l'agitation des journées sanglantes de juin 1848 est perçue par Frédéric comme "misérable à côté [...] de la nature éternelle $\aleph^{55}$. Tout n'est cependant qu'un jeu de perspectives: l'événement historique, qui semble capital lorsqu'il est vu à travers le regard des hommes, paraît ne pas avoir d'existence réelle, considéré au point de vue de la Nature éternelle ; mais cette Nature elle-même, envisagée au point de vue d'un Dieu éternel, se révèle sujette aux bouleversements cataclysmiques. Le véritable point de vue sub specie aeternitatis fait apparaître le monde comme mouvementé, accidenté sous une apparence d'uniformité: l'éternité n'est donc qu'une suite ininterrompue d'événements et l'événement constitue l'autre face de la permanence : dire que tout change et que rien ne change revient au même. Flaubert déconstruit, sur le plan humain, comme sur le plan cosmique, l'opposition entre révolution et statu quo. Il transcende la configuration ordinaire du temps - qui postule une dualité entre le muable et l'immuable, l'éternel et le transitoire - et aboutit ainsi à l'intuition d'une indifférenciation vertigineuse.

La révolution de 1848 , bien qu'elle instaure un changement de régime politique, ne semble pas constituer une véritable rupture au sein de l'ordre bourgeois, comme se plaît à le souligner Flaubert dans Bouvard et Pécuchet :

Dans la matinée du 25 février 1848, on apprit à Chavignolles, par un individu venant de Falaise, que Paris était couvert de barricades - et le lendemain, la proclamation de la République fut affichée sur la mairie.

Ce grand événement stupéfia les bourgeois.

Mais quand on sut que la Cour de cassation, la Cour d'appel, la Cour des comptes, le Tribunal de commerce, la Chambre des notaires, l'Ordre des avocats, le Conseil d'État, l'Université, les généraux et M. De La Rochejacquelein lui-même donnaient leur adhésion au Gouvernement provisoire, les poitrines se desserrèrent [...].5

La permanence du consensus bourgeois par-delà la transformation institutionnelle est bien le signe que ce qui se présente comme un « grand événement » politique n'en est pas un. 
31 À la lumière d'un tel constat, Flaubert décèle donc une forme de sagesse chez ceux qui ignorent souverainement les événements historico-politiques, comme c'est le cas parfois de simples paysans. Ainsi écrit-il dans une lettre de 1853 :

J'ai eu aujourd'hui un grand enseignement donné par ma cuisinière. Cette fille, qui a vingt-cinq ans et est Française, ne savait pas que Louis-Philippe n'était plus roi de France, qu'il y avait eu une république, etc. Tout cela ne l'intéresse pas (textuel). Et je me regarde comme un homme intelligent! Mais je ne suis qu'un triple imbécile. C'est comme cette femme qu'il faut être. ${ }^{57}$

L'écrivain lui-même, à l'école de sa cuisinière, revendiquera un semblable désintérêt pour les vicissitudes de la politique. La retraite de Croisset à ce titre est un des moyens par lesquels Flaubert se distancie des événements contemporains : en 1868, il écrit à Georges Sand "Je vis absolument comme une huitre. Mon roman est le rocher qui m'attache, et je ne sais rien de ce qui se passe dans le monde. $»^{58}$ En 1876, il écrit à sa nièce, « j'ignore tellement ce qui se passe dans le monde que jeudi dernier, seulement, j'ai appris la chute du ministère ! événement dont je me fiche comme de colin-tampon " ${ }^{59}$. Mais le voyage en Orient a été sans doute l'expérience la plus complète d'un décentrement vis-à-vis des événements qui agitent la vie politique française; en mai 1850, Flaubert écrivait d'Égypte :

Quant à nous, nous n'avons pas reçu de nouvelles d'Europe depuis la fin de janvier dernier. Voilà en effet quatre grands mois que nous vivons sur le Nil, ne voyant que ruines, crocodiles et fellahs. Ce n'est pas le moyen d'être fort en politique ni de se tenir au courant du mouvement social. [...] Tu vois que nous nous foutons complètement de tout ce qui se passe et que nous vivons comme de grands égoïstes, aspirant à pleins poumons le bon air chaud des tropiques, contemplant le ciel bleu, les palmiers et les chameaux, buvant du lait de buffle, fumant dans de longues pipes et dormant le nez aux étoiles..$^{60}$

Il serait sans doute caricatural de voir en Flaubert un homme parfaitement indifférent aux évolutions de la société de son temps. D'autres lettres témoignent d'un intérêt et d'une attention aigus pour les problèmes politiques contemporains: la démission de Mac-Mahon en janvier 1879, remplacé par Jules Grévy lui semble «un événement considérable $»^{61}$, et la crise du 16 mai 1876 l'avait mis en « fureur $»^{62}$ contre Mac-Mahon pendant de longs mois. Il n'en reste pas moins que Flaubert tend à définir la posture de l'artiste dans une forme de refus de l'événement, considéré comme un concept fondamentalement bourgeois: "ce paysan, écrit-il à propos du fermier des Commanville, est moins stupide que les trois quarts des bourgeois, lesquels sont toujours à s'agiter d'après le journal. ${ }^{63}$

\section{«Tout fait événement pour qui sait frémir » 64 : immobilité et révolutions infimes}

$\mathrm{Au}$ plan personnel, la "manière spéciale de vivre ${ }^{65}$ qu'invente l'artiste consiste à éviter toute forme d'événement : "Je vis d'une façon farouche et extravagante [...], sans un événement, sans un bruit. C'est le néant objectif, complet. $»^{66}$ écrit-il en 1858 . Plus précisément, il s'agit de se rendre insensible à l'éventualité de l'événement, comme l'indique cette lettre de 1846 :

Il me semble que je suis maintenant dans un état inaltérable. [...] Quand je pense à tout ce qui peut survenir, je ne vois pas ce qui pourrait me changer; j'entends le fond, la vie, le train ordinaire des jours; et puis je commence à prendre une 
habitude du travail dont je remercie le ciel. Je lis ou j'écris régulièrement de huit à dix heures par jour ; et si l'on me dérange, j'en suis tout malade. ${ }^{67}$ Flaubert à une éthique stoïcienne, plus ou moins dégradée, comme l'indique une lettre de 1848: "Moi, cher Ernest (est-ce stoïcisme ou insensibilité de brute ou engourdissement d'âne sur lequel on a tant tapé que rien n'y fait plus), il me semble que tout ce qui peut survenir maintenant m'est fort égal, je deviens philosophe. $»^{68}$ L'écrivain, semblable au sage stoïcien, serait donc capable de s'enfermer dans la citadelle intérieure de sa raison, véritable acropole sur laquelle les événements n'ont aucune prise $^{69}$. Comme l'explique Claude Romano, le stoïcisme constitue «un refoulement de l'événement hors des marges de la philosophie ${ }^{70}$ au nom d'un idéal éthique d'ataraxie. Il semble bien en effet que Flaubert, en se fixant pour tâche «de retrouver pour l'esthétique ce que le stoïcisme avait inventé pour la morale $»^{11}$, entreprenne lui aussi de reléguer l'événement hors de sa vie d'artiste et de son œuvre. À l'instar des stoïciens, Flaubert s'est entraîné à la pratique d'exercices spirituels visant à se rendre invulnérable à l'événement par le travail sur les représentations intérieures et l'imagination: "Avant la mort de mon père et de ma sœur, j'avais assisté à leur enterrement, et quand l'événement est arrivé, je le connaissais. ${ }^{72}$

Mais malgré cet effort philosophique pour atténuer la portée des événements sur son âme et pour s'organiser une vie à l'écart de tout événement, l'écrivain ne parvient pas à s'enfermer dans l'enceinte d'un monde intérieur inaltérable : l'image de la forteresse alterne alors avec celle du "marais dormant »: "Mon existence, comme un marais dormant, est si tranquille que le moindre événement y tombant y cause des cercles innombrables, et la surface ainsi que le fond est longtemps avant de reprendre sa sérénité $! »^{73}$ La métaphore du marais donne une nouvelle signification au calme plat que l'écrivain se plaît à entretenir dans son existence. En effet, l'eau dormante d'un quotidien tranquille est aussi une eau que tout met en éveil. Dès lors,le refus de l'événement a pour contrepartie une hypersensibilité au « moindre événement » ou au micro-événement. L'indifférence recherchée permet le surgissement des différences.

C'est d'ailleurs la raison pour laquelle Flaubert s'écarte d'une certaine sensibilité schopenhauerienne qui est celle de Maupassant, lorsque celui-ci lui écrit: "les événements ne sont pas variés ${ }^{74}$. Schopenhauer ne voit en effet dans les événements qu'une représentation illusoire du devenir, destinée à occulter l'empire de la Volonté comme éternelle répétition du même. Comme le résume Clément Rosset à propos de la pensée schopenhauerienne, "les événements passés étant identiques (quant à leur essence) aux événements présents et aux événements futurs, il n'est rien, dans le cours du temps, qui puisse s'interpréter comme symptôme d'écoulement ${ }^{75}$; "rien ne devient ni n'advient jamais $»^{76}$. Or Flaubert récuse cette philosophie de l'absurde et la disqualification de l'événement qu'elle implique, comme le montre sa réponse à Maupassant: " "Les événements ne sont pas variés." Cela est une plainte réaliste, et d'ailleurs qu'en savez-vous? Il s'agit de les regarder de plus près. " ${ }^{77}$ Loin de constituer l'essence des événements, la répétition n'en caractérise selon Flaubert que la surface : l'événement singulier peut donc être redécouvert à condition d'opérer une conversion du regard vers l'infime et le minuscule. Telle est peut-être la leçon des Pygmées dans La Tentation de saint Antoine : «[...] sous l'arbre qui nous abrite, des révolutions se passent, sans troubler le moineau qui chante dans son feuillage ni les fourmis qui se traînent sur son écorce. $\aleph^{78} \mathrm{Il}$ s'agit pour l'écrivain de saisir ces révolutions minimes, en changeant l'échelle de son regard. À la contemplation macrocosmique, qui était celle de 
Fontainebleau, où la révolution géologique écrasait la révolution politique, succède donc la contemplation microcosmique. Jacques Rancière, analysant le passage où Bouvard et Pécuchet lisent des récits de la Révolution de 1789 dans le calme heureux de leur jardin, a bien montré comment le récit flaubertien supprimait toute hiérarchie entre l'événement de la grande Histoire et l'événement infime de la vie naturelle :

Quelquefois, arrivait un flot d'hommes en bonnet rouge, inclinant au bout d'une pique une tête décolorée, dont les cheveux pendaient. La haute tribune de la Convention dominait un nuage de poussière, où des visages furieux hurlaient des cris de mort. Quand on passait au milieu du jour, près du bassin des Tuileries, on entendait le heurt de la guillotine, pareil à des coups de mouton.

Et la brise remuait les pampres de la tonnelle, les orges mûrs se balançaient, par intervalles un merle sifflait. ${ }^{79}$

L'écriture de Flaubert consiste, selon Jacques Rancière, précisément à "passer pardessous le monde des choses identifiées » et à « les saisir au point où elles se dissolvent en événements purs, c'est-à-dire insignifiants, non pris dans une chaîne de fins et de moyens $»^{80}$. L'événement extraordinaire peut ainsi surgir dans le monde infraordinaire :

J'oubliais un événement extraordinaire : tantôt, comme j'étais seul, j'ai fait un tour jusque dans le potager !!! le temps était splendide. Je suis resté en contemplation devant la nature, et j'ai été pris d'un tel attendrissement pour le petit veau qui était couché près de sa mère sur les feuilles sèches éclairées par le soleil, que je l'ai baisé au front, le susdit veau !81

La contemplation de la Nature permet d'apercevoir sur un fond d'immutabilité apparente des variations et des métamorphoses qui constituent de véritables aventures sensibles, comme lorsque Flaubert en Orient s'extasie devant les bigarrures de la mer :

je suis resté tout seul à regarder la mer. Jamais je n'oublierai cette matinée-là. J'en ai été remué comme d'une aventure. Le fond de l'eau était plus varié de couleurs, à cause de toutes ces coquilles, coquillages, madrépores, coraux, etc., que ne l'est au printemps une prairie couverte de primevères. Quant à la couleur de la surface de la mer, toutes les teintes possibles y passaient, chatoyaient, se dégradaient de l'une sur l'autre, se fondaient ensemble, depuis le chocolat jusqu'à l'améthyste, depuis le rose jusqu'au lapis-lazuli et au vert le plus pâle..$^{82}$

La féerie semble se retrouver dans l'expérience sensible, qui bouleverse le sujet " comme une aventure »: Emma se plaignait de ne jamais « changer de décor "; mais si l'on y regarde de plus près, le décor ne cesse de changer.

41 Si le récit flaubertien se construit en partie sur les ruines de l'événement historique et personnel, c'est qu'il promeut une nouvelle vision de l'aventure : ce qui advient aux êtres, les remue et les transforme, ce sont de petites perceptions, de petites émotions, dont ils peuvent reconnaître à l'occasion que "c'est ce qu'[ils ont] eu de meilleur ". Deux adolescents qui se risquent dans un bordel un jour d'été, offrent des bouquets de fleurs aux prostituées et s'enfuient, gênés, avant d'avoir consommé quoi que ce soit: telle est «l'aventure commune» de Frédéric et Deslauriers, qui «fit toute une histoire » à Nogent, parce qu'on les a vus sortir du bordel. Mais tel est peut-être l'idéal du récit flaubertien - cette histoire à laquelle le lecteur n'aura pas entièrement accès ( Ils se la contèrent prolixement, chacun complétant les souvenirs de l'autre » ${ }^{83}$ ), histoire sur rien, dans laquelle les petits riens sont des événements. 


\section{NOTES}

1. Gérard Genette, Nouveau discours du récit, Paris, Seuil, 1983, « Poétique », p. 14.

2. Lettre de Flaubert à Louise Colet, 2 mai 1852, Correspondance, édition électronique par Yvan Leclerc et Danielle Girard, Centre Flaubert, Université de Rouen, en ligne : https://flaubert.univrouen.fr/jet/public/correspondance/trans.php ?corpus $=$ correspondance\&id $=9921$.

3. Jean Rousset, "Madame Bovary ou le livre sur rien », Forme et signification, Paris, Corti, 1964, p. 133.

4. Voir le chapitre synthétique de Marc Courtieu, «De Flaubert au naturalisme », dans Événement et roman : Une relation critique, Amsterdam, Rodopi, 2012, « Faux Titre ».

5. Fernand Braudel repérait au XIX ${ }^{\mathrm{e}}$ siècle certains précurseurs de l'histoire de la longue durée, parmi lesquels Jules Michelet, Fustel de Coulanges, Leopold von Ranke ou encore Jacob Burckhardt. Voir Fernand Braudel, "Histoire et sciences sociales : la longue durée ", Annales. Économies, Sociétés, Civilisations, $13^{\mathrm{e}}$ année, $\mathrm{n}^{\circ} 4$, 1958. p. 725-753. Plusieurs critiques ont déjà souligné la proximité de Flaubert avec l'historiographie de la longue durée. Citons notamment Gisèle Séginger, Une poétique de l'histoire, Strasbourg, Presses universitaires de Strasbourg, 2000 ; et Nicolas Bourguinat, "Quelques réflexions d'historiens sur Salammbô. Savoirs en récit I", Anne Herschberg Pierrot (dir.), Paris, Presses universitaires de Vincennes, 2010, p. 35-61, p. 45.

6. Flaubert, Madame Bovary, Euvres complètes, éd. Claudine Gothot Mersch, Paris, Gallimard, Bibliothèque de la Pléiade, 2013, t. III, p. 204. (Nous soulignons.)

7. Ibid.

8. Ibid., p. 213.

9. Balzac, Eugénie Grandet, La Comédie humaine, t. III, dir. Pierre-Georges Castex, Paris, Gallimard, Bibliothèque de la Pléiade, 1976, p. 1025.

10. Manuscrits de Madame Bovary, vol. I, fo271, édition électronique sous la direction d'Yvan Leclerc, Centre Flaubert, Université de Rouen, En ligne : https://www.bovary.fr/folio_visu.php? mode $=$ sequence $\&$ folio $=165 \&$ org $=2 \&$ zoom $=50 \&$ seq $=129 \& \mathrm{ppl}=3$.

11. Ibid., vol. I, $\mathrm{f}^{\circ} 84 \mathrm{v}^{\circ}$, en ligne: https://www.bovary.fr/folio_visu.php? mode $=$ sequence $\&$ folio $=1463 \&$ org $=2 \&$ zoom $=50 \&$ seq $=70 \& p p l=3$.

12. Ibid., vol. I, fo 270 , en ligne: https://www.bovary.fr/folio_visu.php? mode $=$ sequence $\&$ folio $=163 \&$ org $=2 \&$ zoom $=50 \&$ seq $=129 \& \mathrm{ppl}=3$.

13. Baudelaire, «L'irréparable », Les Fleurs du mal, CEuvres complètes, t. I, éd. Claude Pichois, Paris, Gallimard, Bibliothèque de la Pléiade, 1975, p. 55.

14. Gautier, Histoire de l'art dramatique en France depuis vingt-cinq ans, Bruxelles, Hetzel, 1858-1859, 6 vol., t. II, p. 175.

15. Balzac, Argow le Pirate, CEuvres de jeunesse, Paris, Michel Lévy, 1866, p. 113.

16. L'Éducation sentimentale, éd. Stéphane Dord-Crouslé, Paris, Flammarion, GF, 2001, p. 143-144.

17. Hannah Arendt, La Nature du totalitarisme, Paris, Payot, 1990, p. 55.

18. Voir Bouvard et Pécuchet, éd. Stéphanie Dord-Crouslé, Paris, GF Flammarion, 2008, chap. III, p. 133-134. Je ne reviendrai pas en détail sur ce passage, analysé par Gisèle Séginger, dans « La réécriture de Cuvier: la création du monde entre savoir et féerie ", Revue Flaubert, $\mathrm{n}^{\circ} 13$, Stéphanie Dord-Crouslé (dir.), 2013, en ligne : https://flaubert.univ-rouen.fr/revue/article.php ? id $=154$.

19. Bouvard et Pécuchet, éd. cit., p. 133.

20. Pour un approfondissement du lien entre l'imaginaire catastrophiste et la féerie, voir Jörg Dünne, Die Katastrophische Feerie: Geschichte, Geologie un Spectakel in der modernen französcichen Literatur, Konstanz, Konstanz University Press, 2016.

21. Bouvard et Pécuchet, éd. cit., p. 134. 
22. Ibid., p. 144-145.

23. Madame Bovary, éd. cit., p. 238.

24. L'Éducation sentimentale, éd. cit., p. 318.

25. Madame Bovary, éd. cit., p. 415.

26. Salammbô, Euvres complètes, éd. Claudine Gothot Mersch, Paris, Gallimard, Bibliothèque de la Pléiade, 2013, t. III, p. 777.

27. Madame Bovary, éd. cit., p. 189.

28. Ibid., p. 198.

29. Pour le rapprochement de l'épisode du bal avec le conte de Perrault, voir Serge Zenkine, Madame Bovary et l'oppression réaliste, Presses de l'université Blaise Pascal, Clermont-Ferrand, 1996, p. 114

30. Manuscrits de Madame Bovary, éd. électronique citée, vol. I, f॰213, en ligne: https:// www.bovary.fr/folio_visu.php $?$ mode $=$ sequence \&folio $=1225 \&$ org $=2 \&$ zoom $=50 \&$ seq $=108 \& p p l=3$. 31. Voir ce que Georges Poulet définit comme l'expérience du «temps-abîme » chez Flaubert dans Études sur le temps humain 1, Monaco, Éditions du Rocher, 1976, p. 357.

32. Flaubert, Novembre, Euvres de jeunesse, éd. Claudine Gothot Mersch et Guy Sagnes, Paris, Gallimard, Bibliothèque de la Pléiade, 2001, p. 782.

33. Timothy Unwin, "Emma Bovary et "les jours où l'on a vécu deux existences" ", Bulletin Flaubert-Maupassant, n² 23, « Madame Bovary, 150 ans après », 2008, p. 309-317, p. 317.

34. Madame Bovary, éd. cit., p. 292.

35. Ibid., p. 293.

36. Madame Bovary, éd. cit., p. 400.

37. Pierre Nora, «L'événement monstre », Communications, $n^{\circ}$ 18, Paris, Seuil, 1972, p. 162-172, p. 165.

38. Lettre à Edma Roger des Genettes, 12 janvier 1878, Correspondance, éd. électronique citée, en ligne : https://flaubert.univ-rouen.fr/jet/public/correspondance/trans.php ? corpus $=$ correspondance $\& \mathrm{id}=12914$.

39. Lettre à Maxime Du Camp, 13 novembre 1879, ibid., en ligne : https://flaubert.univ-rouen.fr/ jet/public/correspondance/trans.php ?corpus =correspondance\&id =13422.

40. Sur le rapport de Flaubert à la pensée évolutionniste, nous nous permettons de renvoyer à notre chapitre, "L'évolutionnisme comme "vrai positivisme" : une pensée du devenir ", dans L'Âme et le Corps chez Flaubert, Paris, Classiques Garnier, 2014, p. 267-282.

41. Jean-Paul Sartre, Qu'est-ce que la littérature ?, Paris, Gallimard, « Folio essais », 1985, p. 226.

42. Michel Crouzet, «L'Éducation sentimentale et le genre historique », L'Éducation sentimentale et la confusion des genres. Ironie-Histoire-Roman, Paris, Eurédit, 2017, p. 1-51.

43. Victor Brombert, «L'Éducation sentimentale: articulations et polyvalence », La production du sens chez Flaubert, Claudine Gothot Mersch (dir.), Paris, Union générale d'éditions, 1975, p. 55-69.

44. L'Éducation sentimentale, éd. cit., p. 376.

45. Claude Romano, L'événement et le monde, Paris, PUF, 1998, «Épiméthée », p. 44.

46. Ibid., p. 43.

47. Ibid., passim.

48. L'Éducation sentimentale, éd. cit., p. 392.

49. Ibid., p. 396.

50. Journal dans lequel, du reste, il s'empresse de célébrer le pseudo-événement de l'opération du pied-bot, dans un style qui substitue au réel sa réécriture féérique : «l'opération [...] s'est pratiquée comme par enchantement ».

51. Ibid., p. 217.

52. Voir Gisèle Séginger, Flaubert. Une Poétique de l'histoire, op. cit.

53. L'Éducation sentimentale, éd. cit., p. 436. 
54. NAF 17607, fo147, transcription Éric Le Calvez, La Production du descriptif. Exogenèse et endogenèse de L'Éducation sentimentale, Amsterdam, New-York, Rodopi, n²24, 2002, «Faux Titre ", p. 242.

55. L'Éducation sentimentale, éd. cit., p. 438.

56. Bouvard et Pécuchet, éd. Stéphanie Dord-Crouslé, Paris, GF-Flammarion, 2008, p. 217.

57. Lettre de Flaubert à Louise Colet, 30 avril 185, Correspondance, éd. électronique citée, en ligne : https://flaubert.univ-rouen.fr/jet/public/correspondance/trans.php ?

corpus $=$ correspondance $\&$ id $=10007$.

58. Lettre de Flaubert à George Sand, 9 septembre 1868, Correspondance, éd. électronique citée, en ligne : https://flaubert.univ-rouen.fr/jet/public/correspondance/trans.php ? corpus $=$ correspondance $\& \mathrm{id}=11278$.

59. Lettre de Flaubert à sa nièce Caroline, 9 décembre 1876, Correspondance, éd. électronique citée, en ligne: https://flaubert.univ-rouen.fr/jet/public/correspondance/trans.php ? corpus $=$ correspondance $\&$ id $=12664$.

60. Lettre de Flaubert à Emmanuel Vasse de Saint Ouen, 17 mai 1850, Correspondance, éd. électronique citée, en ligne: https://flaubert.univ-rouen.fr/jet/public/correspondance/ trans.php ?corpus =correspondance\&id $=9826$.

61. Lettre de Flaubert à Edma Roger des Genettes, 11 février 1879, Correspondance, éd. électronique citée, en ligne: https://flaubert.univ-rouen.fr/jet/public/correspondance/ trans.php ?corpus $=$ correspondance\&id $=13163$.

62. Lettre de Flaubert à Léonie Brainne, 12 novembre 1877, Correspondance, éd. électronique citée, en ligne $:$ https://flaubert.univ-rouen.fr/jet/public/correspondance/trans.php ?act =i\&manus $=0$. 63. Lettre de Flaubert à sa nièce Caroline, 9 décembre 1876, Correspondance, éd. électronique citée, en ligne :

URL : $\quad$ https://flaubert.univ-rouen.fr/jet/public/correspondance/trans.php ? corpus $=$ correspondance $\&$ id $=12664$.

64. Jean Follain, D'après tout, Paris, Gallimard, 1967, p. 52.

65. Lettre de Flaubert à Paule Sandeau, 7 août 1859, Correspondance, éd. électronique citée, en ligne : https://flaubert.univ-rouen.fr/jet/public/correspondance/trans.php? corpus $=$ correspondance $\& i d=10362$.

66. Lettre de Flaubert à Ernest Feydeau, 19 décembre 1858, Correspondance, éd. électronique citée, , en ligne: https://flaubert.univ-rouen.fr/jet/public/correspondance/trans.php ? corpus $=$ correspondance $\&$ id $=10333$.

67. Lettre de Flaubert à Maxime Du Camp, mai 1846, Correspondance, éd. électronique citée, en ligne : https://flaubert.univ-rouen.fr/jet/public/correspondance/trans.php ? corpus $=$ correspondance $\&$ id $=9658 \&$ mot $=\&$ action $=M$.

68. Lettre de Flaubert à Ernest Chevalier, 3 octobre 1848, Correspondance, éd. électronique citée, en ligne: URL : https://flaubert.univ-rouen.fr/jet/public/correspondance/trans.php ? corpus =correspondance\&id $=9780$.

69. Sur le rapport de Flaubert au stoïcisme, voir les notices «Stoïcisme » et « Marc-Aurèle » du Dictionnaire Flaubert, Gisèle Séginger (dir.), Paris, Champion, 2017.

70. Claude Romano, L'Aventure temporelle, Paris, Presses universitaires de France, 2010, p. 17.

71. Lettre à Louise Colet, 24 avril 1852, Correspondance, éd. électronique citée, en ligne : https:// flaubert.univ-rouen.fr/jet/public/correspondance/trans.php ?corpus =correspondance\&id $=9920$. 72. Lettre à Louise Colet, 21 octobre 1846, Correspondance, éd. électronique citée, en ligne : https://flaubert.univ-rouen.fr/jet/public/correspondance/trans.php ?

corpus $=$ correspondance $\&$ id $=9715$.

73. Lettre à Louise Colet, 14 août 1853, Correspondance, éd. électronique citée, en ligne : https:// flaubert.univ-rouen.fr/jet/public/correspondance/trans.php?

corpus $=$ correspondance $\& \mathrm{id}=10032$. 
74. Voir la citation par Flaubert du propos de Maupassant dans sa lettre du 9 ? août 1878, Correspondance, éd. électronique citée, en ligne: https://flaubert.univ-rouen.fr/jet/public/ correspondance/trans.php ?corpus =correspondance\&id =13011.

75. Clément Rosset, Écrits sur Schopenhauer, Paris, Presses universitaires de France, 2001, p. 193.

76. Ibid., p. 140.

77. Lettre de Flaubert à Maupassant, 9 ? août 1878, Correspondance, éd. électronique citée, en ligne : https://flaubert.univ-rouen.fr/jet/public/correspondance/trans.php? corpus $=$ correspondance $\&$ id $=13011$.

78. Flaubert, La Tentation de saint Antoine (version de 1849), Euvres complètes, t. II, dir. Claudine Gothot Mersch, Paris, Gallimard, Bibliothèque de la Pléiade, 2013, p. 487.

79. Bouvard et Pécuchet, éd. cit., p. 175.

80. Jacques Rancière, "Puissances de l'infime ", Flaubert : éthique et esthétique, Anne Herschberg Pierrot (dir.), Paris, Presses universitaires de Vincennes, 2012, p. 29-44, p. 31.

81. Lettre de Flaubert à sa nièce Caroline, 26 octobre 1871, Correspondance, éd. électronique citée, , en ligne: https://flaubert.univ-rouen.fr/jet/public/correspondance/trans.php ? corpus =correspondance $\&$ id $=11782$.

82. Lettre de Flaubert à Louis Bouilhet, 2 juin 1850, Correspondance, éd. électronique citée, en ligne : https://flaubert.univ-rouen.fr/jet/public/correspondance/trans.php ? corpus $=$ correspondance $\& i d=9827$.

83. L'Éducation sentimentale, éd. cit., p. 551-552.

\section{RÉSUMÉS}

Pour tenter de comprendre la place de l'événement dans la pensée flaubertienne, cet article tente de replacer l'écrivain dans les controverses du XIX ${ }^{\mathrm{e}}$ siècle, qui opposent, au plan de la biologie ou de l'histoire, des représentations continuistes (évolution) ou discontinuistes du temps (révolution). En examinant différentes occurrences, dans l'œuvre de Flaubert, du mot ou de l'idée d'événement - qu'elles relèvent de la vie privée ou collective -, on propose de suivre à la trace une pensée qui tout à la fois récuse, démystifie et redéfinit ce qui fait événement dans la Nature, mais aussi dans l'histoire collective et dans l'existence individuelle.

In order to understand what is an event according to Flaubert, we shall set his work in the context of $19^{\text {th }}$ century biological and historical controversies, which confront two different representations of time: the continuity of evolution on the one hand, and the discontinuity of revolution on the other. By examining various occurrences of the word and idea of event in the Flaubertian corpus - whether applying to private or collective life -, we propose to outline Flaubert's reflection as he rejects, demystifies and redefines what is traditionally labelled as an event, in the natural world as well as in collective history and individual existence.

\section{INDEX}

Mots-clés : catastrophe, féerie, stoïcisme, Schopenhauer, socio-biologie

Keywords : catastrophy, fairy tale, stoicism, Schopenhauer, socio-biology 
AUTEUR

JULIETTE AZOULAI

Laboratoire LISAA (EA 4120), Université Gustave Eiffel 